\title{
Contaminação de alimentos pela migração de componentes de embalagens: Casos de ocorrência
}

\author{
Food contamination by migration of packaging components: Cases of occurrence \\ Contaminación de alimentos por migración de componentes de empaque: Casos de ocurrencia
}

\author{
Andressa Pires de Andrade \\ ORCID: https://orcid.org/0000-0001-7966-4253 \\ Universidade Federal Fluminense, Brasil \\ E-mail: andressapiresandrade@ hotmail.com \\ André Luis Rodrigues de Souza \\ ORCID: https://orcid.org/0000-0003-2220-7857 \\ Universidade Federal do Rio de Janeiro, Brasil \\ E-mail: rodrigues_andreluis@yahoo.com.br \\ Jessica Raquel Sales Carvalho De Souza \\ ORCID: https://orcid.org/0000-0002-7141-2753 \\ Universidade Federal Fluminense, Brasil \\ E-mail: jessicasales@id.uff.br \\ Nathália Ramos de Melo \\ ORCID: https://orcid.org/0000-0002-9533-506X \\ Universidade Federal Fluminense, Brasil \\ E-mail: nathaliarm1@gmail.com
}

\begin{abstract}
Resumo
Obter alimentos seguros e de qualidade são as exigências fundamentais do consumidor no momento da compra. A segurança dos alimentos vem ganhando destaque dia após dia, e a responsabilidade em oferecer alimentos inócuos é função de todos os elos da cadeia produtiva, inclusive do setor de embalagens e quanto aos equipamentos que entram em contato com o alimento. O presente trabalho teve como objetivo realizar um levantamento de dados e informações sobre contaminação de alimentos devido à migração de componentes das embalagens e equipamentos, e analisar os motivos que tem acarretado esta migração. O trabalho apresentou uma abordagem qualitativa, baseado em uma pesquisa bibliográfica em artigos e publicações da legislação de embalagens. A partir da análise de sete relatos apresentados observou-se que um dos motivos que tem se destacado em acarretar a migração de compostos para os alimentos é a negligência no uso desses materiais por parte das empresas fabricantes de embalagens e pelos consumidores. Logo pode-se considerar que os setores de embalagens e equipamentos para alimentos precisam buscar um equilíbrio nos seguintes pontos: melhorar a compatibilidade entre material e alimento, controlar fatores como armazenamento e tempo de contato, levar informação completa ao consumidor através dos rótulos e orientar sobre as instruções de uso adequadas para cada tipo de material. Uma combinação entre todos esses fatores juntamente com uma fiscalização adequada pelos órgãos responsáveis irá inibir reduzindo os casos de contaminação de alimentos por migração de compostos prejudiciais a saúde.
\end{abstract}

Palavras-chave: Segurança dos alimentos; Toxicidade; Consumidores; Migração de compostos.

\begin{abstract}
Obtaining safe and quality food is the fundamental requirement of the consumer at the time of purchase. Food safety is gaining prominence day after day, and the responsibility to offer innocuous food is a function of all links in the production chain, including the packaging sector and the equipment that comes into contact with the food. The present work aimed to carry out a survey of data and information on food contamination due to the migration of components of packaging and equipment, and to analyze the reasons that have caused this migration. The work presented a qualitative approach, based on a bibliographic search on articles and publications of the packaging legislation. From the analysis of seven reports presented, it was observed that one of the reasons that has stood out in causing the migration of compounds for food is the negligence in the use of these materials by packaging manufacturers and consumers. Therefore, it can be considered that the packaging and food equipment sectors need to seek a balance in the following points: to improve the compatibility between material and food, to control factors such as storage and contact time, to bring complete information to the consumer through the labels and to advise on instructions for use for each type of material. A combination of all these factors together with an adequate inspection by Organs responsible agencies will inhibit reducing the cases of food contamination by migration of compounds harmful to health.
\end{abstract}

Keywords: Food safety; Toxicity; Consumers; Migration of compounds. 


\begin{abstract}
Resumen
La obtención de alimentos inocuos y de calidad es el requisito fundamental del consumidor en el momento de la compra. La seguridad alimentaria está cobrando protagonismo día a día, y la responsabilidad de ofrecer alimentos inocuos es función de todos los eslabones de la cadena productiva, incluido el sector del envasado y los equipos que entran en contacto con el alimento. El presente trabajo tuvo como objetivo realizar un relevamiento de datos e información sobre la contaminación de los alimentos por la migración de componentes de envases y equipos, y analizar las razones que han provocado esta migración. El trabajo presentó un enfoque cualitativo, basado en una búsqueda bibliográfica de artículos y publicaciones de la legislación de envases. A partir del análisis de siete informes presentados, se observó que una de las razones que se ha destacado en provocar la migración de compuestos para alimentos es la negligencia en el uso de estos materiales por parte de fabricantes de envases y consumidores. Por tanto, se puede considerar que los sectores de envasado y equipamiento alimentario necesitan buscar un equilibrio en los siguientes puntos: mejorar la compatibilidad entre material y alimento, controlar factores como el tiempo de almacenamiento y contacto, llevar información completa al consumidor a través de las etiquetas y asesorar sobre instrucciones de uso para cada tipo de material. Una combinación de todos estos factores junto con una adecuada inspección por parte de los organismos responsables inhibirá la reducción de los casos de contaminación alimentaria por migración de compuestos nocivos para la salud.
\end{abstract}

Palabras clave: Seguridad alimentaria; Toxicidad; Consumidores; Migración de compuestos.

\title{
1. Introdução
}

\section{Uma embalagem para Alimentos}

Os pioneiros em embalagens de alimentos foram os egípcios, sendo um dos primeiros a usar o papel, havendo referências ao uso do papel para empacotar alimentos (incluindo vegetais e temperos). Entretanto, em tempos mais antigos, materiais naturais estavam disponíveis e eram usados para embalagens que incluíam folhas, peles de animais, cascas de coco e de outros vegetais secas. Posteriormente, cestos de juncos, caixas de madeira, barris de madeira, sacos de tecido, etc. entraram em uso, bem como vasos de cerâmica e recipientes de armazenamento de água. (Gupta \& Dudeja, 2017).

O homem precisou sair de um local fixo para procurar alimentos e condições melhores para a sua sobrevivência, e com isso foi necessário que ele tivesse meios para transportar água e comida em suas viagens de exploração. Dessa necessidade, surgiram as primeiras embalagens rudimentares, feitas com crânio e estômago de animais, chifres ocos, grandes conchas e folhas de plantas (Sousa et al., 2012).

Com o passar do tempo e o conhecimento de novas técnicas, começaram a surgir embalagens artesanais mais elaboradas, confeccionadas de argila, vidro e fibras, tigelas de madeira, cestas de fibras naturais, bolsas de pele de animais, entre outros exemplos que fizeram parte de uma segunda fase de formas e técnicas de embalagem (Gupta \& Dudeja, 2017).

As funções básicas de uma embalagem são: conter, conservar, proteger e viabilizar o transporte dos produtos, porém, com o desenvolvimento do comercio e do auto serviço, foram incorporadas outras funções, tais como informar o consumidor, comunicar-se com ele e vender os produtos a partir de um design e visual que o atraia (Brasil, 2002; Abre, 2020; Berk, 2018).

A embalagem deve oferecer proteção para o alimento dos perigos que ocorrem durante a manipulação, movimentação, estocagem e transporte do mesmo, mantendo as características físicas, químicas, microbiológicas e organolépticas dos produtos.

Hoje as embalagens podem desempenhar funções de interação com o alimento e com o ambiente sendo as funções ativas e/ou inteligentes, não sendo somente uma barreira e completamente inerte. Uma embalagem ativa pode interagir deliberadamente com o alimento de forma desejável objetivando manter sua qualidade, alterar alguma característica e estender sua validade comercial. Já uma embalagem inteligente, estas interagem, monitorando as condições do alimentos, transpondo informações sobre o produto ou sobre as condições de estocagem que afetam sua qualidade, validade comercial e segurança. (Madhusudan et al., 2018; Schaefer \& Cheung, 2018; Sharma et al., 2020) 


\section{Contaminação de alimentos através da migração de componentes}

As embalagens possuem como uma de suas funções principais a proteção e conservação do alimento, impedindo que este seja contaminado por agentes externos, porém, a própria embalagem pode se tornar fonte de contaminação dos produtos alimentícios. Dessa forma, o material utilizado na embalagem deve ser compatível com o produto que estará em contato com ele, evitando que a embalagem seja um veículo de contaminação (Muncke, 2016; McCombie \& Biedermann, 2019; Poças, 2018).

Os materiais usados não são completamente inertes e qualquer substância que seja transferida da embalagem ou do equipamento para o produto pode ocasionar alterações nas características dos alimentos e torná-lo impróprio para consumo (Muncke, 2016; McCombie \& Biedermann, 2018; Poças, 2018).

De acordo com a ANVISA, "a transferência de componentes do material em contato com alimentos para estes produtos, devido a fenômenos físico-químicos" é chamada de migração (Brasil, 2001).

A RDC 91/2001 (Brasil, 2001), regulamenta quais são os critérios gerais das embalagens e equipamentos que estejam em contato direto com alimentos. São eles:

a) As embalagens e equipamentos devem ser fabricados em conformidade com as boas práticas de fabricação para que, nas condições normais ou previsíveis de emprego, não produzam migração para os alimentos de componentes indesejáveis, tóxicos ou contaminantes em quantidades tais que superem os limites máximos estabelecidos;

b) Não ocasionem uma modificação inaceitável na composição dos alimentos ou nas características sensoriais dos mesmos;

c) Não representem um risco para a saúde humana.

A migração ocorre devido à interação entre o material da embalagem e o alimento acondicionado, e esse processo pode ser potencializado devido alguns fatores, como as características físico-químicas do alimento, tais como pH e percentual de lipídios, tempo de contato, a temperatura de armazenamento, relação superfície de contato/volume de alimento, entre outros (Melo, 2007; Dias, 2016).

A migração de componentes para os alimentos pode ocorrer de maneiras diferentes dependendo do material. Desse modo, para assegurar o controle necessário sobre estes componentes, foram elaboradas leis que devem ser seguidas pela indústria de embalagens (McCombie \& Biedermann, 2018; Poças, 2018).

Os componentes utilizados nos materiais destinados a entrar em contato com alimentos devem estar inclusos nas listas positivas, que são listas de "substâncias que provaram ser fisiologicamente inócuas em ensaios com animais e cujo uso está autorizado para a fabricação de materiais que entrarão em contato com alimentos” (Brasil, 2001).

Os componentes presentes nas listas positivas não apresentaram riscos quando como usados em embalagens primárias, não ultrapassando o limite de migração total (LMT), limite de migração específica (LME) e limite de composição (LC), estabelecidos por lei, quando existentes.

A RDC 91/2001 (Brasil, 2001) traz as seguintes definições:

Migração total ou global: é a quantidade de componentes transferida dos materiais em contato com alimentos ou seus simulantes, nas condições usuais de emprego, elaboração e armazenamento ou nas condições equivalentes de ensaio.

Migração específica: é a quantidade de um componente não polimérico particular de interesse toxicológico transferida dos materiais de contato com alimentos para os alimentos ou seus simulantes, nas condições equivalentes de ensaio.

Limite de migração total ou global (LMT): é a quantidade máxima admissível de componentes de material em contato com alimentos, transferida aos simulantes sob as condições de ensaio.

Limite de migração específica (LME): é a quantidade máxima admissível de um componente específico do material em contato com alimentos, transferida aos simulantes, nas condições de ensaio. 
Limite de composição (LC): é a quantidade máxima permitida de im componente particular de interesse toxicológico no material em contato com alimentos.

Simulante: é um produto que imita o comportamento de um grupo de alimentos que tem características semelhantes.

\section{Metodologia}

O presente trabalho é uma pesquisa de abordagem qualitativa, baseado em uma pesquisa bibliográfica baseada em artigos, dissertações, revistas eletrônicas e publicações da legislação de embalagens, dados esses em circulação nacional e internacional, sobre a contaminação de alimentos através da migração de substâncias e equipamentos utilizados em alimentos.

$\mathrm{O}$ trabalho foi dividido em duas partes, na qual a primeira define e relata a origem das embalagens, explica sobre a contaminação de alimentos devido a migração de componentes e informa sobre as principais legislações para materiais em contato com alimentos vigentes até o presente momento.

A segunda parte apresenta casos onde mostram os perigos da migração de substâncias da embalagem ou equipamento para o produto, colocando em risco a saúde do consumidor. Foram selecionados aleatoriaamente 7 (sete) casos com o objetivo de verificar se os materiasi utilizados na indústria de embalagens são adequados para o contato direto com o alimento e se não respeitados os limites de migração estabelecidos na legislação.

\section{Casos de ocorrência}

\section{Caso 1: Migração de plastificantes do filme de poli (cloreto de vinila) (pvc) para alimentos gordurosos}

Em um estudo realizado por Bazílio et al. (2014) no Rio de Janeiro-Br, pelo Instituto Nacional de Controle de qualidade em Saúde, Fundação Oswaldo Cruz (INCQS/Fiocruz), foram analisadas trinta e sete amostras de filme flexível de poli (cloreto de vinila), conhecido como PVC, para a determinação da migração específica dos plastificantes ftalato de di-(2etil-hexila) - DEHP e adipato de di-(2-etil-hexila)-DEHA, da embalagem para alimentos gordurosos, utilizando-se simulante. As amostras foram recolhidas de diferentes marcas e provenientes de diversos supermercados do estado.

Estes plastificantes não possuem uma ligação química forte com o polímero de PVC, e dependendo das condições, podem migrar do plástico para o alimento que está em contato com o filme. De forma geral, a literatura mostra que os maiores problemas ocorrem quando o alimento envolvido no PVC possui elevado teor de gordura ou se ele passa por um processo de aquecimento, acarretando uma maior taxa de migração dos plastificantes (Bhunia et al., 2013; Guerreiro et al., 2018).

A resolução $\mathrm{n}^{\circ}$ 17, de 17 de março de 2008, publicada pela ANVISA, regulamenta o limite de migração específica para estes dois componentes, onde o LME do DEHP é de $1,5 \mathrm{mg} \cdot \mathrm{kg}^{-1}$ do simulante e para o DEHA são de $18 \mathrm{mg} \cdot \mathrm{kg}^{-1}$ do simulante.

O estudo obteve como resultado que, do total de amostras ensaiadas, 95\% (35 amostras) apresentaram resultados insatisfatórios no ensaio de migração da embalagem para alimentos gordurosos para pelo menos um dos plastificantes DEHP e/ou DEHA.

Esse resultado mostra que é necessário um maior controle na produção de filmes PVC e conscientização sobre o uso do DEHP e DEHA, pois pesquisas realizadas em ratos de laboratório sobre essas substâncias revelaram que os ftalatos têm potencial cancerígeno, além de provocarem danos ao sistema reprodutivo, por sua atuação como disruptor endócrino. A Agência Internacional para Pesquisa em Câncer - IARC elevou o grau de risco do DEHP, classificando o mesmo no grupo 2B (possível agente carcinogênico ao homem). 


\section{Caso 2: Migração de compostos fluorados para embalagem de fast food}

Um estudo realizado por pesquisadores do Instituto Silent Spring, nos EUA, e publicado na revista científica Environmental Science \& Technology Letters em 2017, com o título "Fluorinated Compounds in U.S. Fast Food Packaging" (em português, Compostos Fluorados em Embalagens para Fast Food nos EUA) apresentou mais um motivo para que as pessoas evitem o consumo de lanches industrializados (Schaider et al., 2017).

As embalagens resistentes à gordura utilizadas em fast-food para acondicionar pizzas, batatas-fritas e hambúrgueres podem conter substâncias químicas fluoradas potencialmente prejudiciais à saúde. Os compostos químicos perfluorados (PFCs), também conhecidos como PFASs, são químicos sintéticos altamente persistentes e quando em contato com o alimento e ingeridos pelo consumidor, podem trazer modificações no DNA e nos processos de replicação celular do indivíduo (Blum et al., 2015; Shoeib et al., 2016).

Estas substâncias estão associadas a distúrbios de fertilidade, doenças da tireoide, puberdade precoce, câncer e diabetes. E as crianças, um dos principais públicos das redes de fast-food são ainda mais prejudicadas, pois seu sistema imunológico ainda está em formação, tornando-as mais suscetíveis a contaminação por essas substâncias.

Foram analisadas 407 (quatrocentos e sete) embalagens de papel e cartolina de 27 (vinte e sete) cadeias de fastfood americanas. O resultado encontrado não foi animador, pois $56 \%$ dos pacotes de sobremesas continham PFCs, assim corno $38 \%$ das embalagens de hambúrgueres e sanduíche e $20 \%$ das caixinhas de cartonado para batata-frita. Apenas os copos de papel estavam livres dessas substâncias.

Os autores do estudo também alertam que os compostos fluorados são altamente resistentes à degradação e quando descartados em aterros sanitários podem migrar para a água no subsolo, acarretando em prejuízos no abastecimento de água potável (Blum et al., 2015; Schaider et al., 2017).

\section{Caso 3: Bisfenol pode desregular hormonios da tireoide mesmo em dose baixa}

O bisfenol A (BPA) é utilizado principalmente na produção de policarbonato e em vernizes epoxi. Pelas propriedades conferidas ao material por essa substância, muitos itens de plástico são produzidos com BPA,como brinquedos, copos infantis (chuquinhas), talheres descartáveis, garrafões retornáveis (20 litros) de água mineral, além de também estar presente em vernizes utilizados para revestimentos de embalagens metálicas para alimentos (Anvisa, 2014). Essa substância pode ser transferida para os alimentos caso ocorra mudança brusca na temperatura, como no aquecimento ou congelamento da embalagem (Toledo, 2017).

Desde janeiro de 2012 alguns países, inclusive o Brasil, optaram por proibir a importação e fabricação de mamadeiras que contenham BPA, devido alguns estudos que taram duvidas sobre a segurança na utilização dessa substância, considerando a reposição e susceptibilidade dos indivíduos usuários deste produto. Para as aplicações, o BPA ainda é permitido e a legislação estabelece limite máximo de migração específica desta substância para o alimento (Anvisa,2014).

Entretanto, Wisniewski et al., 2015 mostrou que mesmo em doses inferiores à considerada segura pelas agências reguladoras, o BPA pode ser um perigo para a tireoide.

Trinta ratos machos foram aleatoriamente divididos em três grupos de 10 (dez) ratos cada, recebendo diariamente dosagens de BPA.

Os autores concluiram que mesmo em uma dosagem considerada desprovida de toxicidade reprodutiva, a exposição ao BPA compromete a produção e funcionalidade dos espermatozoides e perturba o eixo hipotalâmico-hipofisáriogonadal, resultando em estado de hipogonadismo, condição em que as gônadas não produzem quantidades adequadas de hormônios sexuais. Portadores dessa disfunção apresentam redução da libido, prejuízos na fertilidade, perda de massa muscular, ganho de massa gorda, entre outros sintomas. 


\section{Caso 4: Estudo aponta presença de bisfenol a no organismo de adolescentes}

Um estudo feito pela Universidade de Exeter, no Reino Unido e publicado no British Medical Journal tinha como objetivo examinar se as mudanças no estilo de vida e dieta de adolescentes poderiam ter um impacto na presença de BPA em seus organismos (Galloway et al., 2018).

A pesquisa foi realizada com 94 (noventa e quatro) estudantes com idade entre 17 (dezessete) e 19 (dezenove) anos de escolas no sudoeste do Reino Unido. Os participantes receberam orientações para reduzir seu contato com o BPA evitando alimentos processados e embalados em fontes conhecidas por conter essa substância por um período de sete dias.

Foram coletadas amostras de urina em frascos livres de BPA e ao final do estudo, dos noventa e quatro estudantes avaliados, $86 \%$ ainda tinham vestígios de BPA em seu organismo depois que a semana terminou.

O estudo também chamou a atenção para onipresença dessa substância em tantos produtos e a dificuldade em identificar nos rótulos a presença do mesmo.

"Nossos alunos que seguiram a dieta livre de BPA relataram que seria difícil segui-la a longo prazo, já que o químico não é rotulado na embalagem. Eles tiveram dificuldade em identificar alimentos sem BPA", disse Tamara Galloway, professora de Ecotoxicologia da Universidade de Exeter e uma das lideres do estudo.

\section{Caso 5: Benzofenona em materiais de embalagem de papelão e os fatores que influenciam sua migração para o alimento}

A benzofenona é amplamente usada como um foto iniciador para tintas e vernizes que são curados com luz ultravioleta (UV). Os fotoiniciadores são responsáveis pela absorção da radiação UV, acelerando a secagem da tinta. Os fotoiniciadores, apesar de serem fundamentais na formulação, são considerados substâncias com alto potencial de migração do material de embalagem para os alimentos e bebidas".

A benzofenona pode estar presente em embalagens de alimentos cartonados com resíduo de tintas curadas por UV e no papel cartão feito de material reciclado, pois há a possibilidade dessa substância continuar no material, mesmo que a própria embalagem reciclada não tenha sido impressa com tintas curadas por UV contendo benzofenona.

Um artigo publicado pela Food Additives and Contarninants verificou a presença de benzofenona em embalagens de papelão e a consequente migração dessa substância para diversos tipos de alimentos acondicionados em diferentes condições (Anderson \& Castle, 2003).

Foram recolhidas 350 (trezentos e cinquenta) amostras de embalagens de papelão do varejo. Um total de 207 (duzentos e sete) amostras, correspondente a 59\% das embalagens, não possuíam quantidades significativas de benzofenona. Os $41 \%$ restantes continham certa quantidade da substância presente na embalagem.

A partir das 143 (cento e quarenta e três) amostras de embalagens que continham benzofenona, um total de 71 (setenta e uma) amostras foram então selecionadas aleatoriamente e o alimento em si foi analisado. Foi detectada a presença de benzofenona em $72 \%$ dos alimentos.

O mais alto índice de benzofenona foi encontrado no chocolate embalado em contato direto com papel cartão, em temperatura ambiente e com urna área de contato alta. Isso ocorreu porque o chocolate é um alimento rico em gordura e a benzofenona é lipossolúvel.

Além de avaliar se houve a migração da substância para o produto, o estudo também analisou quais eram os fatores que influenciavam a migração da benzofenona. Os fatores analisados foram os regimes de contato (contato direto e indireto) e o tipo de armazenamento (refrigerado/congelado e temperatura ambiente). 
Os resultados mostraram que os efeitos em minimizar a migração do contato indireto e do armazenamento de baixa temperatura são cumulativos. Assim, houve urna redução de seis vezes na migração para contato indireto em comparação com o contato direto, uma redução de seis vezes para armazenamento refrigerado/congelado comparado com armazenamento em temperatura ambiente e redução de quarenta vezes para as duas condições combinadas (armazenamento refrigerado/congelado e contato indireto).

\section{Caso 6: Migração de melamina para alimentos}

A melamina é uma substância química que serve para produzir uma resina dura e resistente, com vasto uso industrial. Essa substância é aprovada para uso na fabricação de alguns utensílios de cozinha, tigelas, produtos de plástico, revestimentos industriais, entre outros (Lu et al., 2009).

Em uma sessão de perguntas e respostas publicada pelo United States Food and Drug Administration (FDA) são respondidas algumas questões em relação à melamina e a migração dessa substância para alimentos (FDA, 2019).

Primeiramente, o FDA esclarece que a melamina pode ser utilizada na confecção de embalagens para produtos alimentícios, mas não é aprovada para adição direta em alimentos para humanos ou animais comercializados nos EUA.

Com este estudo verificou-se que a melamina não migra dos utensílios de cozinha para a maioria dos alimentos. Os testes mostraram a migração de apenas 3 (três) de 19 (dezenove) pratos e xícaras disponíveis comercialmente, para alimentos ácidos, sob condições extremas, ou seja, a comida era mantida na mesa a $160^{\circ} \mathrm{F}\left(71,1^{\circ} \mathrm{C}\right)$ por duas horas. Quando ajustado para as condições reais de uso, a migração foi inferior a 10 partes por bilhão de melamina. Isso é 250 vezes menor do que o nível de melamina aceitável, ou seja, bem abaixo do nível de risco.

No entanto, quando alimentos altamente ácidos, como molho de tomate, suco de laranja ou vinagre são aquecidos a temperaturas acima de $71^{\circ} \mathrm{C}$, a quantidade de melamina que migra do plástico pode aumentar. Por esse motivo, alimentos e bebidas não devem ser aquecidos em utensílios à base de melamina'em fornos de micro-ondas.

Para determinar o nível de melamina nos alimentos que não representa risco para a saúde do consumidor, foi realizada uma avaliação de risco por cientistas do Centro de Segurança Alimentar e Nutrição Aplicada e pelo Centro de Medicina Veterinária da FDA, através de revisão de literatura científica sobre a toxicidade da melamina e estudos em animais que forneceram informações importantes para o resultado do estudo.

Os níveis de migração são relativamente baixos na maioria dos casos, mas a exposição constante pode causar alguns efeitos à saúde. Em caso de contaminação por melamina acima dos níveis observados na avaliação de risco da FDA, as pessoas contaminadas podem desenvolver pedras nos rins e insuficiência renal, e em alguns casos, até a morte. Os sinais de intoxicação por melamina podem incluir irritabilidade, sangue na urina, pouca ou nenhuma urina, sinais de infecção nos rins e / ou pressão alta.

\section{Caso 7: Chumbo é detectado em tampa de três marcas de iogurte}

Um estudo realizado no Centro de Tecnologia de Embalagem do Instituto de Tecnologia de Alimentos (Ital), apresentou alta concentração de chumbo em tampas de iogurtes (Kiyataka et al., 2014).

Para o estudo, foram recolhidas no mercado de Campinas, em São Paulo-Br, 15 (quinze) amostras de iogurtes acondicionados em frascos de polietileno de alta densidade. Três amostras de embalagens de fabricantes diferentes foram estocadas com a tampa para baixo, com objetivo de estudar se haveria a migração dos elementos provenientes das tampas para o alimento. 
Nos testes com as três amostras de tampas utilizadas em embalagens para iogurte de 900 rnL, observou-se a migração de chumbo para o simulante que representa aproximadamente $0,01 \%$ do teor total de chumbo presente nas tampas avaliadas, índice superior ao limite estabelecido pela ANVISA.

Os autores informaram que, apesar de o contato entre a tampa e o alimento ser mínimo, não há como negar o risco de ocorrer à migração do chumbo para o iogurte, principalmente no manuseio. Um exemplo é o transporte deitado do produto ou estocado de ponta cabeça.

Para Kiyataka et al. (2014) é uma falha grave do setor de embalagens e dos órgãos fiscalizadores a comercialização de tampas com alto teor de chumbo, que podem contaminar tanto o consumidor, quanto o ambiente após serem descartadas.

O uso de aditivos e substâncias com arsênio, cádmio e, principalmente, chumbo ou matérias-primas contaminadas com esses elementos na produção de embalagem, indicam a necessidade de uma melhor conscientização por parte dos fabricantes de embalagens e dos usuários, fiscalização e uma legislação ambiental.

\section{Considerações Finais}

A partir da análise dos sete casos apresentados observou-se que o principal motivo que tem acarretado a migração de componentes para os alimentos é a grande negligência no uso desses materiais por parte das empresas fabricantes de embalagens e a forma de utilização pelos consumidores.

O setor de embalagens e equipamentos para alimentos precisa buscar a compatibilidade entre material e alimento, controlar fatores como tipo de armazenamento e tempo de contato, levar informação ao consumidor através dos rótulos quanto as instruções de uso adequado para aquele tipo de material.

Um equilíbrio entre todos esses fatores juntamente com uma fiscalização adequada dos órgãos responsáveis irá diminuir consideravelmente inibir, reduzindo os casos de contaminação de alimentos por migração de componentes tóxicos das embalagens.

\section{Sugestões para Trabalhos Futuros}

Análise "in loco" quanto a contaminação de produtos que estejam em contato com materiais de embalagem, usando simulantes de alimentos assim com os próprios alimentos.

Identificação cromatográfica do bisfenol presente em alimentos envasados com embalagens plásticas.

\section{Referências}

Agência Nacional de Vigilância Sanitária - ANVISA. (2014). Perguntas e Respostas sobre Materiais em contato com alimentos. http://sindlitoralnorters.com.br/images/anvisa_agencia_nacional_de_vigilancia.pdf.

Anderson, W. A. C., \& Castle, L. (2003). Benzophenone in cartonboard packaging materials and the factors that influence its migration into food. Food Additives and Contaminants, 20(6), 607-618.

Associação Brasileira De Embalagens - ABRE. (2019). https://www.abre.org.br/.

Bazilio, F. S., Barros, G. L. M. \& Abrantes, S. M. P. (2015). Controle Sanitário de Filmes Flexíveis de PVC Comercializados no Estado do Rio de Janeiro. Revista Visa em debate, 3(3), 78-84.

Berk, Z. (2018). Food packaging. Food Process Engineering and Technology, 625-641, 2018. 
Bhunia, K., Sablani, S. S., Tang, J., \& Rasco, B. (2013). Migration of Chemical Compounds from Packaging Polymers during Microwave, Conventional Heat Treatment, and Storage. Comprehensive Reviews in Food Science and Food Safety, 12, 523545 .

Blum, A., Balan, S. A., Scheringer, M., Trier, X., Goldenman, G., Cousins, I. T., Diamond, M., Fletcher, T., Higgins, C., Lindeman, A. E., Peaslee, G., Voogt, P., Wang, Z., \& Weber, R. (2015). The Madrid Statement on Poly- and Perfluoroalkyl Substances (PFASs). Environmental Health Perspectives, 123(5), 107-111.

Brasil.(1999). Portaria n ${ }^{\circ}$ 177, de 04 de março de 1999. Aprova o regulamento técnico - Disposições gerais para embalagens e equipamentos celulósicos em contato com os alimentos. Diário Oficial [da] República Federativa do Brasil. http://portal.anvisa.gov.br/documents/33916/390501/PORTARIA_177.pdf/67e5bd39-b018-418c-ab62-bb0a5f956aeb.

Brasil. (2001). Agência Nacional de Vigilância Sanitária. Resolução RDC nº 91, de 11 de maio de 2001. Aprova o regulamento técnico - Critérios gerais e classificação de materiais para embalagens e equipamentos em contato com alimentos. Diário Oficial [da] República Federativa do Brasil. http://portal.anvisa.gov.br/documents/10181/2718 376/(1)RDC_91_2001_COMP.pdf/fb132262-e0a1-4a05-8ff7-bc9334c18ad3.

Brasil. (2002). Agência Nacional de Vigilância Sanitária. Resolução RDC nº 259, de 20 de setembro de 2002. Aprova o regulamento técnico - Critérios gerais e classificação de materiais para embalagens e equipamentos em contato com alimentos. Diário Oficial [da] República Federativa do Brasil. http://bvsms.saude.gov.br/bvs/saudelegis/anvisa /2002/rdc0259_20_09_2002.html.

Dias, D. A. B. (2016). Migração de contaminantes de materiais de embalagens. Dissertação em Ciências Farmacêuticas. Portugal: Instituto Superior de Ciências da Saúde Egas Moniz.

FDA - United States Drug and Administration. (2019). Melamine in Tableware: Questions and Answers. https://www.fda.gov/food/chemicals/melamine-tableware-questions-and-answers.

Galloway, T. S., Baglin, N., Lee, B. P., Kocur, A. L., Shepherd, M. H., Steele, A. M. \& Harries, L. W. (2018). An engaged research study to assess the effect of a 'real-world' dietary intervention on urinary bisphenol A (BPA) levels in teenagers. British Medical Journal Open, 8 (2), 1-7.

Geueke, B., Groh, K. \& Muncke, J. (2018). Food packaging in the circular economy: overview of chemical safety aspects for commonly used materials. Journal of Cleaner Production, 193, 491-505.

Guerreiro, T. M., Oliveira, D. N., Melo, C. F. O. R., Lima, E. O. \& Catharino, R. R. (2018). Migration from plastic packaging into meat. Food Research International, 109, 320-324.

Gupta, R. K. \& Dudeja, P. Food packaging. (2017). Food Safety in the 21st Century, 547-553.

Kiytataka, P. H. M., Dantas, S. T. \& Pallone, J. A. L. (2014). Method for assessing lead, cadmium, mercury and arsenic in high density polyethylene packaging and study of the migration into yoghurt and simulant. Food Additives \& Contaminants. Part A. Chemistry, Analysis, Control, Exposure \& Risk Assessment, 31(1), 156-163.

Lu, J., Xiao, J., Yang, D. J., Wang, Z.T., Jiang, D. G., Fang, C. R. \& Yang, J. (2009). Study on migration of melamine from food packaging materials on markets. Biomedical and Environmental Sciences, 22, 104-108.

Madhusudan, P., ChellukurI, N. \& Shivakumar, N. (2018). Smart packaging of food for the 21st century - A review with futuristic trends, their feasibility and economics. Materials Today: Proceedings, 5, 21018-21022.

Majid, I., Nayik, G. A., Dar, S. M., \& Nanda, V. Novel food packaging technologies: Innovations and future prospective. Journal of the Saudi Society of Agricultural Sciences, 17, 454-462.

McCombie, G. \& Biedermann, M. (2019). Migration From Food Contact Materials. Encyclopedia of Food Chemistry, 603608 .

Melo, N. R. (2007). Migração de plastificantes e avaliação de propriedades mecânicas de filmes de poli(cloreto de vinila) para alimentos. Tese em Ciência e Tecnologia de Alimentos. Viçosa: Universidade Federal de Viçosa.

Muncke, J. (2016). Chemical Migration from Food Packaging to Food. Reference Module in Food Sciences, DOI: https://doi.org/10.1016/B978-0-08-100596-5.03311-4, 1-7. 
Poças, F. (2018). Migration From Packaging and Food Contact Materials Into Foods, Reference Module in Food Sciences, DOI: https://doi.org/10.1016/B978-0-08-100596-5.21460-1 1-18.

Risch, S.J. (2009). Food Packaging History and Innovations. Journal of Agricultural and Food Chemistry, 57, 8089-8092.

Schaefer, D. \& Cheung, W.M. Smart Packaging: Opportunities and Challenges. Procedia CIRP, 72, 1022-1027.

Schaider, L. A., Balan, S. A., Blum, A., Andrews, D. Q., Strynar, M. J., Dickinson, M. E., Lunderberg, D. M., Lang, J. R. \& Pleaslee, G. F. (2017). Fluorinated Compounds in U.S. Fast Food Packaging. Environmental Science \& Technologic Letters, 4, 105-111.

Sharma, S., Barkauskaite, S., Jaiswal, A. K. \& Jaiswal, S. (2020). Essential Oils as Additives in Active Food Packaging. Food Chemistry.

Shoeib, T., Hassan, Y., Rauert, C. \& Harner, T. (2016). Poly- and perfluoroalkyl substances (PFASs) in indoor dust and food packaging materials in Egypt: Trends in developed and developing countries. Chemosphere, 144, 1573-1581.

Sousa, L. C. F. S., Sousa, J. S., Borges, M. G. B., Machado, A. V., Silva, M. J. S., Ferreira, R. T. F. V. \& Salgado, A. B. (2012). Tecnologia de embalagens e conservação de alimentos quanto aos aspectos físico, químico e microbiológico. Agropecuária Científica no Semiárido, 8, (1), 19-27.

Wisniewski, P., Romano, R. M., Kizys, M. M. L., Oliveira, K. C., Kasamatsu, T., Giannocco, G., Chiamolera, M. I., Silva, M. R. D. \& Romano, M. A. (2015). Adult exposure to bisphenol A (BPA) in Wistar rats reduces sperm quality with disruption of the hypothalamic-pituitary-testicular axis. Toxicology, 329, 1-9. 